\title{
HYPERBOLIC GROUPS AND COMPLETELY SIMPLE SEMIGROUPS
}

\author{
John Fountain \\ Department of Mathematics, University of York, \\ Heslington, York YO10 5DD, U.K. \\ e-mail : jbf1@york.ac.uk \\ Mark Kambites \\ School of Mathematics \& Statistics, Carleton University, \\ Herzberg Laboratories, 1125 Colonel By Drive, \\ Ottawa, Ontario K1S 5B6, \\ Canada \\ e-mail : mkambite@math.carleton.ca
}

\begin{abstract}
We begin with a brief introduction to the theory of word hyperbolic groups. We then consider four possible conditions which might reasonably be used as definitions or partial definitions of hyperbolicity in semigroups: having a hyperbolic Cayley graph; having hyperbolic Schützenberger graphs; having a context-free multiplication table; or having word hyperbolic maximal subgroups. Our main result is that these conditions coincide in the case of finitely generated completely simple semigroups.
\end{abstract}

This paper is based on a lecture given at the workshop on Semigroups and Languages held at the Centro de Álgebra da Universidade de Lisboa in November 2002. The aim of the paper, as of the talk, is to provide semigroup theorists with a gentle introduction to the concept of a word hyperbolic group (hereafter referred to as simply a 'hyperbolic group'), to discuss recent work of Gilman which makes it possible to introduce a notion of (word) hyperbolic semigroup and then to examine these ideas in the context of completely simple semigroups. The first three sections of the paper are expository while the fourth introduces new results. After briefly discussing Dehn's algorithm, we describe hyperbolic groups in terms of 'slim triangles', and then describe some properties of these groups which we need later in the paper. Hyperbolic groups were introduced by Gromov 
in [14]; readers who want to find out more can also consult [1], Chapter III. $\Gamma$ of [4], [11] and [22]. There are many characterisations of hyperbolic groups in terms of the geometry of Cayley graphs, but none of these extend naturally to semigroups because the algebraic properties of a semigroup are not closely related to the geometric properties of any notion of Cayley graph for a semigroup. However, Gilman [13] recently characterised hyperbolic groups in language theoretic terms; given a group $G$ with finite generating set $A$ and language $L$ over $A$ that projects onto $G$, he associated a language with $G$ called the 'multiplication table relative to $L$ ' of the group, and showed that $G$ is hyperbolic if and only if it has a context-free multiplication table relative to some rational language. This led Duncan and Gilman [9] to modify the definition of multiplication table for the semigroup setting and propose a definition of hyperbolic semigroup; using [13] they showed that for groups this is equivalent to the original definition. We give an account of this work in Section 3.

In Section 4 we examine completely simple semigroups which are hyperbolic in the sense of Duncan and Gilman. By definition, such a semigroup $S$ is finitely generated, and so if $S$ is isomorphic to the Rees matrix semigroup $M(G ; I, \Lambda ; P)$, then the sets $I$ and $\Lambda$ must be finite. Given this, one's first guess turns out to be correct, that is, $S$ is hyperbolic if and only if $G$ is hyperbolic. Moreover, in the case of a completely simple semigroup, the geometry of the Schützenberger graphs of the $\mathscr{R}$-classes is relevant. We show that the semigroup is hyperbolic if and only if each of these graphs is hyperbolic in the geometric sense defined in Section 1.

\section{Hyperbolic Groups}

Combinatorial group theory arose from the study of fundamental groups in the early years of the development of topology. It was given impetus and direction by Dehn's publication in 1912 of three basic decision problems: the word problem, the conjugacy problem and the isomorphism problem. We shall discuss only the word problem, and that only briefly.

We denote the free monoid on a non-empty set $A$ by $A^{*}$, and the free semigroup by $A^{+}$. If $A^{-1}$ is the set of symbols $\left\{a^{-1}: a \in A\right\}$, we define $\left(a^{-1}\right)^{-1}=a$ for all $a \in A$, and for a non-empty word $w=x_{1} \ldots x_{n}$ where $x_{i} \in A \cup A^{-1}$, we define $w^{-1}=x_{n}^{-1} \ldots x_{1}^{-1}$; for the empty word 1 , we put $1^{-1}=1$. A group $G$ is generated by $A$ (or $A$ generates $G$ ) if there is a surjective homomorphism $\varphi:\left(A \cup A^{-1}\right)^{*} \rightarrow G$ from the free monoid $\left(A \cup A^{-1}\right)^{*}$ onto $G$ with $a^{-1} \varphi=(a \varphi)^{-1}$ for all $a \in A$. If there is a 
finite set which generates $G$, then $G$ is finitely generated. We also say that $\varphi:\left(A \cup A^{-1}\right)^{*} \rightarrow G$ is a choice of generators for $G$, and speak of a finite choice of generators when $A$ is finite. When $X$ is a finite set which contains a formal inverse for each of its elements, we say that a surjective homomorphism $\varphi: X^{*} \rightarrow G$ is symmetric finite choice of generators for $G$. One wants to know when two words $u, v$ in $\left(A \cup A^{-1}\right)^{*}$ represent the same element of $G$, that is, when $u \varphi=v \varphi$. Equivalently, one is asking when $\left(u v^{-1}\right) \varphi=1$. A word $r$ that represents the identity in $G$ is called a relator, and there is a corresponding relation " $r=1$ ". Let $R$ be a set of relators and consider the congruence $\sim$ on $\left(A \cup A^{-1}\right)^{*}$ generated by

$$
\{(r, 1): r \in R\} \cup\left\{\left(x x^{-1}, 1\right): x \in A \cup A^{-1}\right\} .
$$

If the congruences $\sim$ and $\operatorname{ker} \varphi$ coincide, then $R$ is called a set of relators for $G$, and the pair $\langle A \mid R\rangle$ is a presentation for $G$. Rather than specifying a relator, one often specifies a relation $u=v$, meaning that $u v^{-1}$ is a relator. The group $G$ is finitely presented if it has a presentation $\langle A \mid R\rangle$ with both $A$ and $R$ finite. We mention the fact that if $A$ and $B$ are finite sets of generators for $G$ and if $G$ has a finite presentation $\langle A \mid R\rangle$, then it has a finite presentation $\langle B \mid S\rangle$ for some set $S$ of words over $B \cup B^{-1}$.

A group $G$ with a finite generating set $A$ has solvable word problem if the set of words over $A$ which represent the identity in $G$ is recursive, that is, there is an algorithm which will decide which words in $\left(A \cup A^{-1}\right)^{*}$ represent the identity and which do not.

Novikov [21], Boone [5] and Britton [6] independently proved that there are finitely presented groups with unsolvable word problem.

On the positive side, there are some presentations which lead to a fast algorithm for solving the word problem. Let $A$ be a finite set of generators for a group $G$; recall that a word in $\left(A \cup A^{-1}\right)^{*}$ is freely reduced if it has no factors of the form $a a^{-1}$ or $a^{-1} a$ with $a \in A$, and that any word may be freely reduced by deleting such factors until none remain. Now suppose that we have a finite list of words $u_{1}, \ldots, u_{n}, v_{1}, \ldots, v_{n}$ such that, for each $i$, the words $u_{i}$ and $v_{i}$ represent the same element of $G$ and $\left|v_{i}\right|<\left|u_{i}\right|$; suppose further that every non-empty freely reduced word that represents the identity of $G$ contains at least one of the $u_{i}$ as a factor. Then we have the following algorithm to solve the word problem for $G$. Given any word $w$, freely reduce it to obtain a word $w^{\prime}$; if $w^{\prime}$ is not empty and does not have a factor $u_{i}$ for any $i$, it does not represent the identity of $G$. If $w^{\prime}$ does have a factor $u_{i}$, replace it by $v_{i}$ and freely reduce the resulting word to obtain $w^{\prime \prime}$. Note that $\left|w^{\prime \prime}\right|<\left|w^{\prime}\right|$. Now repeat the procedure 
starting with $w^{\prime \prime}$ and continue until the procedure can be no longer applied. Then $w$ represents 1 in $G$ if and only if the end result of the procedure is the empty word. Moreover, one can show that the algorithm is very fast. When a group admits this procedure for solving the word problem, we say that it has a Dehn algorithm. The algorithm leads to a Dehn presentation $\left\langle A \mid u_{1} v_{1}^{-1}, \ldots, u_{n} v_{n}^{-1}\right\rangle$ for $G$.

Dehn proved that Fuchsian groups admit Dehn presentations [8]. In the 1950s and 60s, various 'small cancellation' groups were shown to have Dehn presentations (see [20] for an account of this work). One motivation for the introduction of hyperbolic groups was to answer to the question of which groups admit a Dehn presentation. In his groundbreaking work [14], Gromov proved the following result among many other things.

Theorem 1.1. A group is hyperbolic if and only if it admits a (finite) Dehn presentation.

Having explained one of the reasons for studying hyperbolic groups, we now introduce some concepts which allow us to give one definition for this class of groups. First, let $\varphi:\left(A \cup A^{-1}\right)^{*} \rightarrow G$ be a finite choice of generators for $G$. We consider the (right) Cayley graph $C_{\varphi}(G)$ of $G$ relative to $(A, \varphi)$. This is a (labelled) directed graph with vertex set $G$ and an edge (labelled a) from $g$ to $g(a \varphi)$ for each $g \in G$ and $a \in A$. Each edge is given a local metric in which it has unit length, and $C_{\varphi}(G)$ is turned into a metric space by defining the distance $d_{\varphi}(x, y)$ between two points $x, y$ to be equal to the length of the shortest path joining them. When $\varphi$ is understood, we sometimes refer to the 'Cayley graph relative to the generating set $A$ '.

Notice that if $g, h$ are vertices of $C_{\varphi}(G)$ (that is, elements of $G$ ), then $d_{\varphi}(g, h)$ is the length of the shortest word in $\left(A \cup A^{-1}\right)^{*}$ representing $g^{-1} h$. Restricted to $G$, the metric $d_{\varphi}$ is called the word metric with respect to $(A, \varphi)$. We are interested only in finite generating sets $A$, but even in this case the metric on the Cayley graph and the word metric depend on the choice of $A$. However, if $\psi:\left(B \cup B^{-1}\right)^{*} \rightarrow G$ is another finite choice of generators, then the metric spaces $\left(C_{\varphi}(G), d_{\varphi}\right),\left(G, d_{\varphi}\right),\left(C_{\psi}(G), d_{\psi}\right)$ and $\left(G, d_{\psi}\right)$ are closely related as we now explain.

Let $(X, d)$ and $\left(X^{\prime}, d^{\prime}\right)$ be metric spaces. A function $f: X \rightarrow X^{\prime}$ is a quasi-isometry if there are constants $\lambda \geqslant 1, \epsilon \geqslant 0$ and $C \geqslant 0$ such that every point of $X^{\prime}$ lies in the closed $C$-neighbourhood of $f(X)$ and

$$
\frac{1}{\lambda} d(x, y)-\epsilon \leqslant d^{\prime}(f(x), f(y)) \leqslant \lambda d(x, y)+\epsilon
$$

for all $x, y \in X$. 
When there is a quasi-isometry from $X$ to $X^{\prime}$, the spaces are said to be quasi-isometric. Quasi-isometric metric spaces have many geometric properties in common. We also mention that being quasi-isometric is an equivalence relation on the class of metric spaces.

The following are examples of quasi-isometries.

(1) The natural inclusion $\mathbb{Z} \rightarrow \mathbb{R}$ is a quasi-isometry. Take $\lambda=1, \epsilon=0$ and $C=\frac{1}{2}$. Similarly, the natural inclusion $\mathbb{Z}^{n} \rightarrow \mathbb{R}^{n}$ is a quasiisometry.

(2) Let $G$ be a group with finite choice of generators $\varphi:\left(A \cup A^{-1}\right)^{*} \rightarrow G$ as above. Then the inclusion map $G \rightarrow C_{\varphi}(G)$ is a quasi-isometry from $\left(G, d_{\varphi}\right)$ to $\left(C_{\varphi}(G), d_{\varphi}\right)$ with $\lambda=1, \epsilon=0$ and $C=\frac{1}{2}$.

(3) Let $\varphi:\left(A \cup A^{-1}\right)^{*} \rightarrow G$ and $\psi:\left(B \cup B^{-1}\right)^{*} \rightarrow G$ be finite choices of generators for a group $G$. For each member of $B \psi$ choose a word over $A \cup A^{-1}$ representing it, and similarly, for each element of $A \varphi$ choose a word over $B \cup B^{-1}$ which represents it. Let $N$ be the maximum of the lengths of the chosen words. Then the identity map $G \rightarrow G$ is a quasi-isometry from $\left(G, d_{\varphi}\right)$ to $\left(G, d_{\psi}\right)$ and from $\left(G, d_{\psi}\right)$ to $\left(G, d_{\varphi}\right)$ with $\lambda=N, \epsilon=0$ and $C=0$.

We now introduce the ideas of geodesic and hyperbolic metric spaces. Let $x, y$ be points in a metric space $(X, d)$. A geodesic segment from $x$ to $y$ of length $\ell$ is the image of an isometric embedding $i$ from the closed interval $[0, \ell] \subseteq \mathbb{R}$ with $i(0)=x$ and $i(\ell)=y$. Thus $d(i(s), i(t))=|t-s|$ for all $s, t \in[0, \ell]$.

A metric space is a geodesic (metric) space if, for any two points of the space, there is a geodesic segment from one to the other. A (geodesic) triangle in a metric space consists of three points (the vertices) and, for each pair of these points, a choice of geodesic segment joining them. For a positive constant $\delta$, a triangle in a metric space is $\delta$-slim (following the terminology of [4]) if each edge of the triangle is contained in the closed $\delta$-neighbourhood of the union of the other two edges.

A geodesic metric space $X$ is hyperbolic if there is a global constant $\delta$ such that all triangles in $X$ are $\delta$-slim. The name comes from the fact that these spaces are generalisations of classical hyperbolic space $\mathbb{H}^{n}$ which has the slim triangles property with $\delta=2$. Among other examples of hyperbolic metric spaces, we have the following.

(1) A bounded geodesic metric space is hyperbolic because if the distance between any two points in the space is at most $M$, then clearly 
every triangle is $M$-slim.

(2) Any tree can be regarded as a metric space, and it is geodesic because any two points are connected by a shortest path. All triangles are 0 -slim since any edge of a triangle is contained in the union of the other two, so that a tree is a hyperbolic metric space.

An important fact is that hyperbolicity is an invariant of quasi-isometry among geodesic spaces.

Theorem 1.2. Let $X$ and $X^{\prime}$ be quasi-isometric geodesic spaces. Then $X$ is hyperbolic if and only if $X^{\prime}$ is hyperbolic.

A group $G$ with finite choice of generators $\varphi:\left(A \cup A^{-1}\right)^{*} \rightarrow G$ is said to be hyperbolic if the metric space $\left(C_{\varphi}(G), d_{\varphi}\right)$ is hyperbolic. As $\left(C_{\varphi}(G), d_{\varphi}\right)$ is clearly a geodesic space for any finite choice of generators $\varphi:\left(A \cup A^{-1}\right)^{*} \rightarrow G$, to say that $G$ is hyperbolic means that, for some $\delta$, all geodesic triangles in $\left(C_{\varphi}(G), d_{\varphi}\right)$ are $\delta$-slim. In view of the theorem and our remarks above, this is independent of the choice of finite generating set for $G$. Some examples of hyperbolic groups are the following.

(1) Every finite group is hyperbolic because, relative to any finite set of generators, its Cayley graph is bounded.

(2) Every finitely generated free group is hyperbolic because, relative to a free set of generators, its Cayley graph is a tree.

(3) Let $G$ and $H$ be cyclic groups of order 3 with generators $g$ and $h$ respectively. Then the free product $G * H$ is hyperbolic. This can be seen by considering triangles in the Cayley graph relative to the generating set $\{g, h\}$.

The simplest example of a finitely generated group which is not hyperbolic is $\mathbb{Z} \times \mathbb{Z}$. We can see this by considering the Cayley graph relative to the generating set $\{(0,1),(1,0)\}$. For any positive integer $N$, consider the triangle with vertices $(0,0),(N, 0)$ and $(N, N)$; there is a unique geodesic segment joining $(0,0)$ to $(N, 0)$, and a unique geodesic segment joining $(N, 0)$ to $(N, N)$, but there are several joining $(0,0)$ to $(N, N)$. We choose the one, illustrated in Figure 1, passing through $(0, N)$. Then this point is outside the $(N-1)$-neighbourhood of the union of the edges from $(0,0)$ to $(N, 0)$ and from $(N, 0)$ to $(N, N)$. Hence not all triangles are $(N-1)$-slim, and as this is true for every $N$, the Cayley graph (and so the group) is not hyperbolic. 


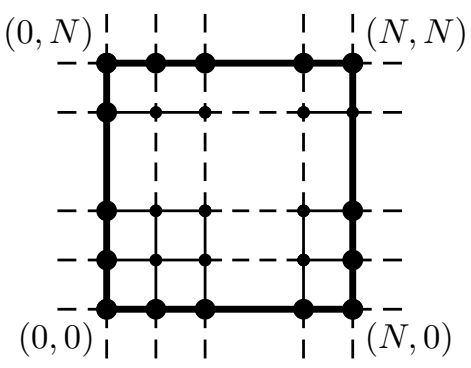

Figure 1

Next, we give a few important properties of hyperbolic groups. Proofs can be found in the books referred to above.

Theorem 1.3. If a group $G$ is hyperbolic, then:

(1) $G$ is finitely presented;

(2) $G$ has solvable word problem;

(3) $G$ has solvable conjugacy problem;

(4) every subgroup of finite index in $G$ is hyperbolic;

(5) every finite extension of $G$ is hyperbolic;

(6) $G$ does not contain a copy of $\mathbb{Z} \times \mathbb{Z}$ as a subgroup;

(7) $G$ has only finitely many conjugacy classes of finite subgroups;

(8) $G$ is biautomatic.

We now describe a property of hyperbolic spaces which will be important for us in Section 4. Let $(X, d)$ be a hyperbolic space and $x, y, z$ be any three points in $X$, and consider a geodesic triangle with these points as vertices. The triangle inequality guarantees that there are unique nonnegative numbers $a, b, c$ such that $d(x, y)=a+b, d(x, z)=a+c$ and $d(y, z)=b+c$. Let $p, q, r$ be the points on the edges from $x$ to $y$, from $x$ to $z$ and from $y$ to $z$ respectively such that $d(x, p)=a, d(x, q)=a$ and $d(y, r)=b$ as shown in Figure 2.

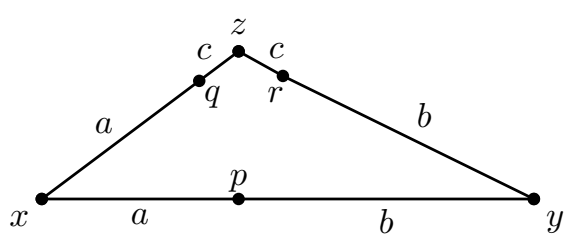

Figure 2

Let $I_{x p}$ denote the geodesic segment from $x$ to $p$ which forms part of the edge 
of the triangle from $x$ to $y$ and so on. There are isometries $\alpha: I_{x p} \rightarrow I_{x q}$, $\beta: I_{y p} \rightarrow I_{y r}$ and $\gamma: I_{z q} \rightarrow I_{z r}$. It is known that the hyperbolicity of the space ensures that there is a global constant $\lambda$ such that for any such triangle we have $d(u, \alpha(u))<\lambda, d(v, \beta(v))<\lambda$ and $d(w, \gamma(w))<\lambda$ for all $u, v, w$ in the domains of $\alpha, \beta, \gamma$ respectively. Following [4] we say that the triangles are $\lambda$-thin. In fact, the property that all geodesic triangles are $\lambda$-thin for some $\lambda \geqslant 0$ characterises hyperbolic spaces (see Proposition 1.17 of Chapter III.H in [4]). Since $X$ is a geodesic space, there is a geodesic (of length less than $\lambda$ ) joining each pair of points which correspond under one of the three isometries $\alpha, \beta$ and $\gamma$.

When the space in question is the Cayley graph of a hyperbolic group and $x, y, z$ are group elements, it is clear that either all the points $p, q, r$ are group elements (vertices of the Cayley graph) or none of them are. Moreover, a group element on a side of the triangle corresponds to another group element under the appropriate isometry. Thus there are two possible configurations which we illustrate schematically in Figure 3 below where the dots represent group elements.
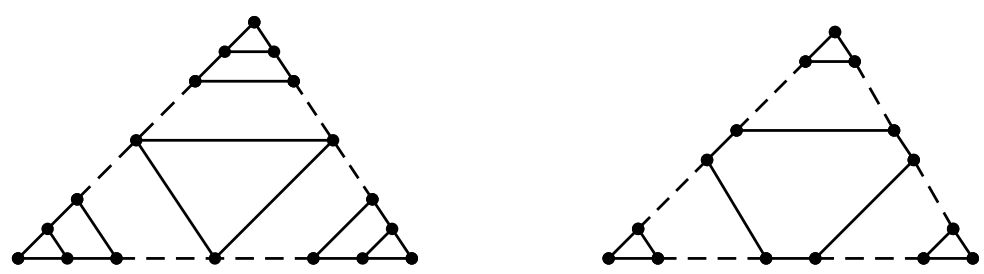

Figure 3

\section{Multiplication Tables of Finitely Generated Groups}

We now turn to Gilman's language theoretic characterisation of hyperbolic groups. We need some preliminaries about formal languages, and we begin by reminding the reader about context-free grammars, languages and rational transductions. For more on formal languages see [3],[15],[17], and for an introduction to the use of formal languages in group theory see [12].

Let $A$ be a finite non-empty set. A context-free grammar over $A$ is a 4-tuple $\Gamma=(V, A, P, S)$ where $V$ is a finite set disjoint from $A, P$ is a finite subset of $V \times(V \cup A)^{*}$ and $S$ is a member of $V$. The elements of $V$ are called variables or non-terminals, those of $A$ are called terminals, those of $P$ are called productions and $S$ is called the start symbol.

If $(T, \alpha) \in P$, we write $T \rightarrow \alpha$. If $\beta, \gamma \in(V \cup A)^{*}$, then we write $\beta \Rightarrow \gamma$ 
if there exist $\delta, \eta$ in $(V \cup A)^{*}$ and a production $T \rightarrow \alpha$ such that $\beta=\delta T \eta$ and $\gamma=\delta \alpha \eta$. The reflexive transitive closure of $\Rightarrow$ is denoted by $\stackrel{*}{\Rightarrow}$. The language generated by $\Gamma$ is defined to be

$$
L(\Gamma)=\left\{w \in A^{*}: S \stackrel{*}{\Rightarrow} w\right\} .
$$

A language $L \subseteq A^{*}$ is context-free if $L=L(\Gamma)$ for some context-free grammar $\Gamma$. We shall be interested in context-free languages in the free semigroup $A^{+}$, so it is worth mentioning the following standard result (where 1 is the empty word).

Lemma 2.1. If $L \subseteq A^{*}$ is context-free, then so are $L \cup\{1\}$ and $L \backslash\{1\}$.

Let $X, Y$ be subsets of a monoid $M$. The product $X Y$ of $X$ and $Y$ is the set $\{x y: x \in X, y \in Y\}$, and the submonoid of $M$ generated by $X$ is denoted by $X^{*}$. The set $\operatorname{Rat}(M)$ of rational subsets of $M$ is the smallest collection of subsets of $M$ satisfying the following two conditions:

(1) every finite subset of $M$ is rational;

(2) if $X, Y \subseteq M$ are rational, then so are $X \cup Y, X Y$ and $X^{*}$.

We obtain the definition of a rational subset of a semigroup $S$ by replacing $M$ by $S$ and modifying (2) by replacing $X^{*}$ by $X^{+}$, the subsemigroup generated by $X$. If $S$ does not have an identity, $S^{1}$ denotes the monoid obtained from $S$ by adjoining an identity. For $X \subseteq S$, we use $X^{*}$ to denote the submonoid of $S^{1}$ generated by $X$. Clearly, $X^{+}=X X^{*}$ and so any rational subset of $S$ is also a rational subset of $S^{1}$.

A (nondeterministic) finite automaton over a monoid $M$ is a finite directed graph (whose vertices are called states) with edges labelled by elements of $M$, a distinguished state called the initial state and a set of states called accept states.

A path in a finite automaton over $M$ is a finite sequence of directed edges such that the terminal vertex of an edge in the sequence is the initial vertex of the next edge in the sequence. The label of a path is the product (in order) of the labels of its edges. A path is successful if it starts at the initial state of the automaton and ends at an accept state. The subset of $M$ accepted by the automaton is the set of labels of successful paths. The significance of this idea is made clear by the following result. For a proof, see, for example, Theorem 2.6 of [12].

Proposition 2.1. A subset of a monoid $M$ is rational if and only if it is accepted by a finite automaton over $M$. 
A rational transduction $\rho: A^{*} \rightarrow B^{*}$ where $A$ and $B$ are finite is a rational subset of the direct product $A^{*} \times B^{*}$. Similarly, one also defines a rational transduction $\rho: A^{+} \rightarrow B^{+}$between finitely generated free semigroups. The rational transductions from $A^{+}$to $B^{+}$are precisely those rational transductions from $A^{*}$ to $B^{*}$ that are contained in $A^{+} \times B^{+}$.

We observe that a homomorphism $\varphi: A^{*} \rightarrow B^{*}$ is an example of a rational transduction since the graph of $\varphi$ is $\{(a, a \varphi): a \in A\}^{*}$. Another example is provided by $\rho_{L}: A^{*} \rightarrow A^{*}$ where $L \subseteq A^{*}$ is rational and $\rho_{L}=\{(w, w): w \in L\}$.

For any binary relation $\rho: X \rightarrow Y$ and subset $L$ of $X$, we put

$$
L \rho=\{y \in Y:(x, y) \in \rho \text { for some } x \in L\} .
$$

The basic properties of rational transductions are collected in the following result. Proofs can be found in Section III.4 of [3] or Section 5 of [12].

Proposition 2.2. Let $A, B, C$ be finite sets and suppose that $\rho: A^{*} \rightarrow B^{*}$, $\sigma: A^{*} \rightarrow B^{*}, \tau: B^{*} \rightarrow C^{*}$ are rational transductions. Then:

(1) $\rho \cup \sigma, \rho \sigma$ (the subset product of $\rho$ and $\sigma$ ) and $\rho^{*}$ are rational transductions;

(2) $\rho^{-1}: B^{*} \rightarrow A^{*}$ and $\rho \circ \tau: A^{*} \rightarrow C^{*}$ are rational transductions;

(3) if $L \subseteq A^{*}$ is rational, then so is $L \rho$;

(4) if $L \subseteq A^{*}$ is context-free, then so is $L \rho$.

Of course, there is a corresponding result for rational transductions between free semigroups. As an immediate corollary of the proposition (and using the example $\rho_{L}$ above), we have the following standard results about rational and context-free languages.

Corollary 2.1. Let $A, B$ be finite non-empty sets, $L$ be a rational language over $A$ and let $\varphi: A^{*} \rightarrow B^{*}$ be a homomorphism.

(1) If $K \subseteq A^{*}$ and $J \subseteq B^{*}$ are rational, then so are $K \cap L, K \varphi$ and $J \varphi^{-1}$.

(2) If $K \subseteq A^{*}$ and $J \subseteq B^{*}$ are context-free, then so are $K \cap L, K \varphi$ and $J \varphi^{-1}$.

We conclude this section by explaining the notion of multiplication table for a finitely generated group and stating Gilman's theorem [13].

Let $\varphi:\left(A \cup A^{-1}\right)^{*} \rightarrow G$ be a finite choice of generators for a group $G$, and let \# be a symbol not in $A$. For $L \subseteq\left(A \cup A^{-1}\right)^{*}$ with $L \varphi=G$, the 
language

$$
M=\{u \# v \# w: u, v, w \in L \text { and }(u v w) \varphi=1\}
$$

over $A \cup\{\#\}$ is called the multiplication table of $G$ relative to $(A, L, \varphi)$.

Theorem 2.1. Let $\varphi:\left(A \cup A^{-1}\right)^{*} \rightarrow G$ be a finite choice of generators for a group $G$. Then $G$ is hyperbolic if and only if there is a rational language $L$ over $A$ with $L \varphi=G$ such that the multiplication table for $G$ relative to $(A, L, \varphi)$ is context-free.

\section{Word Hyperbolic Semigroups}

Theorem 2.1 suggests a way of defining a notion of hyperbolicity for semigroups. This was made explicit by Duncan and Gilman in [9], who proposed the following definition which was subsequently further explored in [16]. A finite choice of generators for a semigroup $S$ is a surjective homomorphism $\varphi: A^{+} \rightarrow S$ where $A$ is a finite alphabet. A choice of representatives for $S$ is a triple $(A, L, \varphi)$ where $\varphi: A^{+} \rightarrow S$ is a finite choice of generators and $L \subseteq A^{+}$is such that $L \varphi=S$. It is a rational choice of representatives if the language $L$ is rational. There are, of course, corresponding notions for monoids. For a word $w=a_{1} \ldots a_{n}$ where $a_{i} \in A$, the reverse of $w$ is $w^{r}=a_{n} \ldots a_{1}$, and $1^{r}=1$. Let \# be a symbol not in $A$. Following [9], we say that $S$ is hyperbolic if, for some finite choice of generators $\varphi: A^{+} \rightarrow S$, there is a rational language $L$ over $A$ with $L \varphi=S$ such that the language $T=\left\{u \# v \# w^{r}: u, v, w \in L\right.$ and $\left.(u v) \varphi=w \varphi\right\}$ is context-free. The language $T$ is called the multiplication table of $S$ relative to $(A, L, \varphi)$, and we say that $S$ is hyperbolic with respect to the choice of generators $\varphi: A^{+} \rightarrow S$.

If $M$ is a monoid, then using a finite choice of monoid generators, we can define $M$ to be 'hyperbolic as a monoid', and, of course, a group may be 'hyperbolic as a semigroup', 'hyperbolic as a monoid' or 'hyperbolic as a group'. However, Duncan and Gilman show that the various notions coincide (see Theorem 3.5 and Corollary 4.3 of 9 ), that is, we have the following result.

\section{Theorem 3.1.}

(1) A monoid $M$ is hyperbolic as a semigroup if and only if it is hyperbolic as a monoid;

(2) A group $G$ is hyperbolic as a semigroup if and only if it is hyperbolic as a group. 
Crucial for this result and of interest in its own right is the following.

Theorem 3.2. If the semigroup $S$ is hyperbolic with respect one finite choice of generators, then it is hyperbolic with respect to every such choice.

It is noted in [9] that finite semigroups are hyperbolic, and it is also shown that the bicyclic monoid $M=\langle a, b \mid a b=1\rangle$ is hyperbolic. The latter shows that, when applied to inverse semigroups, the definition of hyperbolic we are using gives a different class from that arising from a geometric definition of hyperbolic inverse semigroup suggested in [25]. To describe this approach, we need the notion of the Schützenberger graph of an $\mathscr{R}$-class $R$ of a semigroup $S$. These graphs were first defined by Stephen [26] in the context of inverse semigroups, but can be defined quite generally. Let $\varphi: A^{+} \rightarrow S$ be a finite choice of generators for a semigroup $S$, and let $R$ be an $\mathscr{R}$-class of $S$. The Schützenberger graph $\Gamma_{\varphi}(R)$ of $R$ (relative to $\varphi$ ) is a (labelled) directed graph with vertex set $R$ and an edge (labelled $a$ ) from $s$ to $s(a \varphi)$ for each $s \in R$ and each $a \in A$ such that $s(a \varphi) \mathscr{R} s$. Note that $\Gamma_{\varphi}(R)$ is strongly connected; it is made into a metric space (denoted by $\left.\left(\Gamma_{\varphi}(R), d_{\varphi}\right)\right)$ in just the same way that the Cayley graph of a group is. If $\theta: B^{+} \rightarrow S$ is another finite choice of generators, then (just as in the case of Cayley graphs of groups) $\Gamma_{\varphi}(R)$ and $\Gamma_{\theta}(R)$ are quasi-isometric. Again, as in the group case, we may consider the discrete metric space $\left(R, d_{\varphi}\right)$ obtained by restricting the metric to $R$.

In the case where $S$ is an inverse semigroup, one considers a choice of generators of the form $\varphi:\left(A \cup A^{-1}\right)^{+} \rightarrow S$ where, as in the group case, $A$ is an alphabet, $A^{-1}=\left\{a^{-1} \mid a \in A\right\}$ is an alphabet disjoint from $A$ and in one-one correspondence with $A$, and such that for every $a \in A$ we have $a^{-1} \varphi=(a \varphi)^{-1}$. In this case, we observe that whenever $\Gamma_{\varphi}(R)$ contains an edge from $s$ to $t$ labelled $a$, it contains also an edge from $t$ to $s$ labelled $a^{-1}$.

Note that if $G$ is a group, then there is only one $\mathscr{R}$-class, namely $G$ itself, and the Schützenberger graph is the right Cayley graph.

In [25], an inverse semigroup $S$ is defined to be hyperbolic in a way which is equivalent to: $S$ is finitely generated and, for each $\mathscr{R}$-class $R$, the Schützenberger graph of $R$ is hyperbolic and $R$ contains only finitely many $\mathscr{H}$-classes. Clearly, the bicyclic monoid is not hyperbolic in this sense.

\section{Completely Simple Semigroups}

Recall that a semigroup $S$ is completely simple if it has no two-sided ideals other than itself, and it possesses minimal one-sided ideals. It is well known 
(see, for example, Chapter 3 of [18]) that $S$ is completely simple if and only if it is isomorphic to a Rees matrix semigroup $M=M(G ; I, J ; P)$ over a group $G$ where $I, J$ are non-empty sets and $P=\left(p_{j i}\right)$ is a $J \times I$ matrix over $G$ and $M$ is the set $I \times G \times J$ with multiplication given by

$$
(i, g, j)(\ell, h, k)=\left(i, g p_{\ell j} h, k\right) .
$$

We now analyse when such a semigroup is hyperbolic. To be hyperbolic, $S$ must be finitely generated. It is straightforward to show (and is also immediate from a more general result of Ayik and Ruškuc [2]) that this is equivalent to $G$ being finitely generated, and $I$ and $J$ being finite. In other words, the maximal subgroups of $S$ (which are all isomorphic) are finitely generated, and $S$ has finitely many $\mathscr{R}$ - and $\mathscr{L}$-classes. It turns out that adapting the Steinberg [25] definition to completely simple semigroups does give precisely those semigroups that are hyperbolic in the sense of [9].

Following [9], for any semigroup $S$, we define the Cayley graph $C_{\sigma}(S)$ relative to a choice of generators $\sigma: A^{+} \rightarrow S$ to have vertices $S \cup\{*\}$ where $*=1$ if $S$ is a monoid, and $*$ is a symbol not in $S$ otherwise. For each $a \in A$, there is a directed edge with label $a$ from $*$ to $a \sigma$, and, for each $s \in S$, a directed edge with label $a$ from $s$ to $s(a \sigma)$. The distance between two vertices is the length of the shortest undirected path joining the vertices. Then $C_{\sigma}(S)$ is made into a metric space $\left(C_{\sigma}(S), d_{\sigma}\right)$ in just the same way that the Cayley graph of a group is. Again, as in the group case, a change of generators leads to a quasi-isometric space.

Our main result is the following.

Theorem 4.1. Let $S$ be a finitely generated completely simple semigroup isomorphic to the Rees matrix semigroup $M(G ; I, J ; P)$. Then the following are equivalent:

(1) $S$ is hyperbolic;

(2) $G$ is hyperbolic;

(3) for any choice of generators for $S$, the Schützenberger graph of each $\mathscr{R}$-class of $S$ is hyperbolic;

(4) for any choice of generators for $S$, the Cayley graph of $S$ is hyperbolic.

First we establish some notation which will be used throughout the section. Let $S$ be a finitely generated completely simple semigroup with $\mathscr{R}$-classes $R_{1}, \ldots, R_{m}$ and $\mathscr{L}$-classes $L_{1}, \ldots, L_{n}$, and put $R_{i} \cap L_{j}=H_{i j}$. Each $H_{i j}$ is a maximal subgroup of $S$ and we denote its identity by $e_{i j}$. Let 
$R=R_{1}, H=H_{11}$ and $e=e_{11}$. Note that each $R_{i}$ is a subsemigroup with a single $\mathscr{R}$-class.

We now consider the equivalence of (2), (3) and (4).

Let $X \subseteq H$ be a symmetric (that is, closed under inverses) finite set of generators for $H$; we assume that $e_{11} \in X$. Then, as is well known and easy to see, $Y=X \cup\left\{e_{12}, \ldots, e_{1 n}\right\}$ is a generating set for $R$, and $Z=Y \cup\left\{e_{21}, \ldots, e_{m 1}\right\}$ is a generating set for $S$. In each case the choice of generators is given by the inclusion map, so there is no ambiguity in writing $\left(C_{X}(H), d_{X}\right)$ for the Cayley graph of $H$ relative to $(X, \iota)$ regarded as a metric space, and we can use similar notation for the other graphs involved.

Lemma 4.1. The spaces $\left(C_{X}(H), d_{X}\right),\left(\Gamma_{Y}(R), d_{Y}\right),\left(\Gamma_{Z}(R), d_{Z}\right)$ and $\left(C_{Z}(S), d_{Z}\right)$ are quasi-isometric.

Proof. It suffices to consider the discrete spaces $\left(H, d_{X}\right),\left(R, d_{Y}\right),\left(R, d_{Z}\right)$ and $\left(S \cup\{*\}, d_{Z}\right)$. First, note that $e_{1 j} x \in H$ for each $j \in\{2, \ldots, n\}$ and each $x \in X$ so that $e_{1 j} x$ can be expressed as a word over $X$. Choose one such word for each $e_{1 j} x$ and let $M$ be the greatest of the lengths of the chosen words. Next, observe that if $r \in R$, then $r=h e_{1 j}$ for some $h \in H$ and some $j \in\{1, \ldots, n\}$. Hence $d_{Y}(H, r) \leqslant 1$ and so the inclusion map $H \rightarrow R$ is a quasi-isometry from $\left(H, d_{X}\right)$ to $\left(R, d_{Y}\right)$ with $\lambda=M, \epsilon=0$ and $C=1$.

Now consider $\left(R, d_{Z}\right)$ and let $r, s \in R$ be such that there is an edge from $r$ to $s$ labelled by $e_{i 1}$. Suppose that $r \in H_{1 j}$ and put $h_{j i}=e_{1 j} e_{i 1}$. Note that $h_{j i}$ is in $H$ (as is $s$ ) and that $u h_{j i}=u e_{i 1}$ for all $u \in H_{1 j}$. Let $w_{j i}$ be a word over $X$ which represents $h_{j i}$. Then, since $r h_{j i}=r e_{i 1}=s$, there is an edge path from $r$ to $s$ in $\left(R, d_{Y}\right)$ labelled by $w_{j i}$. Let $N$ be the length of the longest of the words $w_{j i}$. Then the identity map $R \rightarrow R$ is a quasi-isometry from $\left(R, d_{Y}\right)$ to $\left(R, d_{Z}\right)$ with $\lambda=N, \epsilon=0$ and $C=0$.

Finally, note that there is no ambiguity in the notation because the $d_{Z}$ metric on $R$ is the restriction to $R$ of the $d_{Z}$-metric on $S$. Thus all we have to do is note that every element of $S \cup\{*\}$ has distance at most 1 from an element of $R$ so that $\left(R, d_{Z}\right)$ and $\left(S \cup\{*\}, d_{Z}\right)$ are indeed quasi-isometric.

If $S$ is isomorphic to the Rees matrix semigroup $M(G ; I, J ; P)$, then $G$ is isomorphic to each maximal subgroup of $S$. Hence, in view of the fact that Cayley graphs of a group (or semigroup) relative to different finite generating sets are quasi-isometric, as are Schützenberger graphs of an $\mathscr{R}$-class of a semigroup relative to different finite generating sets for the semigroup, it follows from Lemma 4.1 and Theorem 1.2 that (2), (3) and 
(4) of Theorem 4.1 are equivalent.

Similarly, the fact that (1) implies (2) in Theorem 4.1 is a consequence of the next proposition.

Proposition 4.1. If $S$ is hyperbolic, then so is $H$.

Proof. Since $S$ is finitely generated we can retain the notation for the $\mathscr{R}$ classes, etc., and since $S$ is hyperbolic, there is a choice of representatives $(A, L, \sigma)$ for $S$ where $L$ is rational and the multiplication table $T$ of $S$ relative to $(A, L, \sigma)$ is context-free.

First, we define a choice of generators for $H$. Put

$$
B=\left\{b_{j a}: a \in A, j \in J\right\}
$$

and define a homomorphism $\rho: B^{+} \rightarrow H$ by $b_{j a} \rho=e_{1 j}(a \sigma) e$. Clearly $B$ is finite. Also $\rho$ maps onto $H$, for if $h \in H$, then $h=\left(a_{1} \sigma\right) \ldots\left(a_{n} \sigma\right)$ for some $a_{1}, \ldots, a_{n} \in A$. Suppose that $a_{k} \sigma \in H_{i(k), j(k)}$. Clearly, $i(1)=1$ and $j(n)=1$ so that, writing $a(i)$ for $a_{i}$ when it occurs as a subscript, we have

$$
\begin{aligned}
h & =\left(a_{1} \sigma\right) \ldots\left(a_{n} \sigma\right) \\
& =e\left(a_{1} \sigma\right) e \cdot e_{1 j(1)}\left(a_{2} \sigma\right) e \cdot e_{1 j(2)}\left(a_{3} \sigma\right) e \ldots e_{1 j(n-1)}\left(a_{n} \sigma\right) e \\
& =\left(b_{1 a(1)} b_{j(1) a(2)} \ldots b_{j(n-1) a(n)}\right) \rho .
\end{aligned}
$$

Next, we find a rational choice of representatives for $H$ over the alphabet $B$. Let $K=H \sigma^{-1}$ and observe that $K=K^{\prime} A^{*} K^{\prime \prime} \cup\left(K^{\prime} \cap K^{\prime \prime}\right)$ where $K^{\prime}=\{a \in A: a \sigma \in R\}$ and $K^{\prime \prime}=\left\{a \in A: a \sigma \in L_{1}\right\}$. Now $K^{\prime}$ and $K^{\prime \prime}$ are finite so that $K$ is rational and hence so is $L \cap K$.

Now define a function $\varphi: K \rightarrow B^{+}$by

$$
\left(a_{1} \ldots a_{n}\right) \varphi=b_{1 a(1)} b_{j(1) a(2)} \ldots b_{j(n-1) a(n)}
$$

where $a_{i} \in A$ and $a_{k} \sigma \in H_{i(k) j(k)}$ as above. We have just seen that $w \varphi \rho=$ $w \sigma$ for all $w \in K$. Since $L \sigma=S$, we have $(L \cap K) \varphi \rho=(L \cap K) \sigma=H$ and thus $(B,(L \cap K) \varphi, \rho)$ is a choice of representatives for $H$. To show that this choice is rational, we show that $\varphi$ is a rational transduction. We define a finite automaton $M$ over the semigroup $A^{+} \times B^{+}$. The state set of $M$ is $\left\{q_{0}\right\} \cup S / \mathscr{L} \cup A$ where we assume the union is disjoint. The initial state is $q_{0}$ and the unique accept state is $L_{1}$. The edge set is

$$
\begin{aligned}
& \left\{\left(q_{0},\left(a, b_{1 a}\right), L_{a \sigma}\right): a \sigma \in R\right\} \\
& \cup\left\{\left(L_{j},\left(1, b_{j a}\right), a\right): a \in A, j \in\{1, \ldots, n\}\right\} \\
& \cup\left\{\left(a,(a, 1), L_{a \sigma}\right): a \in A\right\} .
\end{aligned}
$$


It is straightforward to verify that $M$ accepts the graph of $\varphi$, so that $\varphi$ is rational. By (3) of Proposition 2.2, $(L \cap K) \varphi$ is rational and so we do have a rational choice of representatives.

The multiplication table of $H$ relative to $(B,(L \cap K) \varphi, \rho)$ is

$$
T^{\prime}=\left\{u \# v \# w^{r}: u, v, w \in(L \cap K) \varphi \text { and }(u \rho)(v \rho)=w \rho\right\} .
$$

Thus, since $\varphi \rho=\sigma$, the word $u \# v \# w^{r}$ is in $T^{\prime}$ if and only if $u=u^{\prime} \varphi, v=$ $v^{\prime} \varphi, w=w^{\prime} \varphi$ for some $u^{\prime}, v^{\prime}, w^{\prime} \in L \cap K$ such that $\left(u^{\prime} \sigma\right)\left(v^{\prime} \sigma\right)=w^{\prime} \sigma$, that is,

$$
u^{\prime} \# v^{\prime} \# w^{\prime r} \in T \cap\left(K\{\#\} K\{\#\} K^{r}\right) .
$$

Recall that the reversal $\varphi^{r}$ of $\varphi$ is defined to be

$$
\left\{\left(x^{r}, y^{r}\right) \in K^{r} \times K^{r}:(x, y) \in \varphi\right\},
$$

and that since $\varphi$ is rational, so is $\varphi^{r}$ (see, for example, page 66 of [3]). Next we define a function $\theta: K\{\#\} K\{\#\} K^{r} \rightarrow B^{+}\{\#\} B^{+}\{\#\} B^{+}$by

$$
\left(x \# y \# z^{r}\right) \theta=(x \varphi) \#(y \varphi) \#\left(z^{r} \varphi^{r}\right) .
$$

so that $T^{\prime}=\left(T \cap\left(K\{\#\} K\{\#\} K^{r}\right)\right) \theta$. Clearly, the graph of $\theta$ is $\varphi\{(\#, \#)\} \varphi\{(\#, \#)\} \varphi^{r}$ and so we have that $\theta$ is a rational subset of $(A \cup\{\#\})^{+} \times(B \cup\{\#\})^{+}$. Thus $\theta$ is a rational transduction. Now $T$ is context-free and $K\{\#\} K\{\#\} K^{r}$ is clearly rational so that, in view of Corollary 2.1(2), $T \cap\left(K\{\#\} K\{\#\} K^{r}\right)$ is context-free, and hence, by Proposition 2.2(4), so is $T^{\prime}$. Thus, regarded as a semigroup, $H$ is hyperbolic, and so, by Theorem 3.1 , it is a hyperbolic group.

We now embark on proving that condition (2) of Theorem 4.1 implies condition (1). We start by recalling the following result (see, for example, Corollary 2.20 of Chapter III. $\Gamma$ in [4]).

Proposition 4.2. Let $G$ be a hyperbolic group and $\sigma:\left(A \cup A^{-1}\right)^{*} \rightarrow G$ be a finite choice of generators. Then the set $P$ of words in $\left(A \cup A^{-1}\right)^{*}$ which label geodesics in the Cayley graph $C_{\sigma}(G)$ is a rational language.

We call the set $P$ the language of geodesics of $G$ relative to $\sigma$. The following lemma and its proof are inspired by Section 4 of [13].

Lemma 4.2. Let $\sigma: A^{*} \rightarrow G$ be a symmetric finite choice generators for a hyperbolic group $G$, and let $P$ be the language of geodesics. Then the language

$$
Q=\{u \# v \# w \# x: u, v, w, x \in P \text { and }(u v w x) \sigma=1\}
$$

over $A \cup\{\#\}$ is context-free. 
Proof. First, we note that $C=P\{\#\} P\{\#\} P\{\#\} P$ is a rational subset of $(A \cup\{\#\})^{*}$. We produce a context-free grammar $\mathscr{G}$ such that $Q=L(\mathscr{G}) \cap C$, and hence $Q$ is context-free as desired.

Since $G$ is hyperbolic, there is a non-negative constant $\lambda$ such that all geodesic triangles in the Cayley graph $C_{\sigma}(G)$ are $\lambda$-thin. Define a contextfree grammar $\mathscr{G}$ as follows. The terminal alphabet is $A \cup\{\#\}$ and the set of variables is

$$
V=\left\{X_{z}: z \in(A \cup\{\#\})^{*} \text { and }|z| \leqslant 2 \lambda\right\} .
$$

Let $W=A \cup\{\#\} \cup V$ and extend $\sigma: A^{*} \rightarrow G$ to a homomorphism $\sigma: W^{*} \rightarrow G$ by defining $X_{z} \sigma=z \sigma$ and $\# \sigma=1$.

In this proof we denote the empty word by $\epsilon$; the start symbol of the grammar is $X_{\epsilon}$ and the set of productions consists of all pairs $\left(X_{z}, \alpha\right)$ where $\alpha \in W^{*},|\alpha| \leqslant 7$ and $X_{z} \sigma=\alpha \sigma$.

Clearly, if $X_{z} \stackrel{*}{\Rightarrow} \beta$, then $X_{z} \sigma=\beta \sigma$ and so the grammar generates a context-free language consisting of certain words which are mapped to 1 by $\sigma$. To complete the proof, it is clearly enough to show that $Q \subseteq L(\mathscr{G})$.

Let $u \# v \# w \# x \in Q$ so that $u, v, w, x$ label geodesics in $C_{\sigma}(G)$ and $(u v w x) \sigma=1$. Note that $X_{\epsilon} \rightarrow \epsilon \# \epsilon \# \epsilon \# \epsilon$ is a production, so that certainly $\epsilon \# \epsilon \# \epsilon \# \epsilon \in L(\mathscr{G})$. If some but not all of $u, v, w, x$ are empty, then the words are labels of the sides of a geodesic triangle or a degenerate geodesic triangle in $C_{\sigma}(G)$, and the argument in Section 4 of [13] shows that $u \# v \# w \# x$ is in $L(\mathscr{G})$. Thus we may assume that $u=a_{1} \ldots a_{p}, v=b_{1} \ldots b_{q}, w=c_{1} \ldots c_{r}$ and $x=d_{1} \ldots d_{s}$ with $a_{i}, b_{j}, c_{k}, d_{h}$ being letters in $A$. Then in $C_{\sigma}(G)$ there is a geodesic quadrilateral with vertices $1, u \sigma,(u v) \sigma$ and $(x \sigma)^{-1}$ and geodesic sides labelled $u, v, w$ and $x$. There is at least one geodesic segment joining $(x \sigma)^{-1}$ and $u \sigma$. Consider one such; then this segment is the common side of two geodesic triangles, as illustrated in Figure 4.

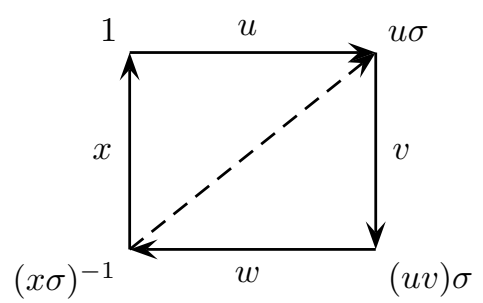

Figure 4

Since each triangle in the diagram is $\lambda$-thin, we can pair the points on 
their perimeters as described at the end of Section 1 in such a way that each pair of matched points is joined by a path of length at most $\lambda$. Joining the paths which meet at the common side of the two triangles gives a matching between points on the sides of the quadrilateral, and matched points are joined by a path of length at most $2 \lambda$. Moreover, a point which is a group element is matched with one or more group elements.

Let $L_{u}, L_{v}, L_{w}, L_{x}$ be the sides of the quadrilateral with labels $u, v, w, x$ respectively in Figure 4 , and let $L$ be the side joining $(x \sigma)^{-1}$ to $u \sigma$. Let $\Delta$ be the triangle with sides $L_{u}, L_{x}$ and $L$, and $\Delta^{\prime}$ be that with sides $L_{v}, L_{w}$ and $L$. Consider a group element $g$ on $L_{u}$. In $\Delta$, the point $g$ is matched with at most one point on $L_{x}$ and at most one point on $L$. If it is matched with a point on $L$, then the latter point is matched with at most one point on $L_{v}$ and with at most one point on $L_{w}$. Thus $g$ cannot be matched with more than one point on a specific side of the quadrilateral.

The matching described can occur in one of several ways. A group element on a particular side may be matched with:

(i) a group element on an adjacent side;

(ii) a group element on an opposite side;

(iii) a group element on an adjacent side and a group element on an opposite side;

(iv) group elements on each of the adjacent sides;

$(v)$ group elements on each of the other three sides.

Which of these occur depends on the nature of the configuration obtained when we put together paths joining matching points in $\Delta$ and $\Delta^{\prime}$ which meet on the side $L$. The configuration is determined by where the central triangle or hexagon in $\Delta$ and that in $\Delta^{\prime}$ meet the common side $L$. This can occur in one of several (actually 16) ways. The following diagrams illustrate three of the possibilities; the assiduous reader can easily draw the remaining ones.

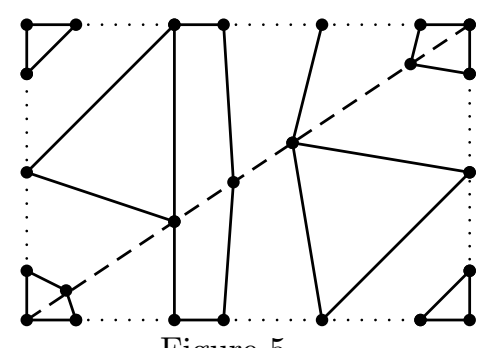

Figure 5 
In Figure 5 both geodesic triangles have central triangles; the central triangle of $\Delta$ meets the common side to the left of the central triangle in $\Delta^{\prime}$. Two of the other possible configurations are illustrated below in Figure 6 . In these diagrams some of the edges and some of the group elements are labelled for later reference.

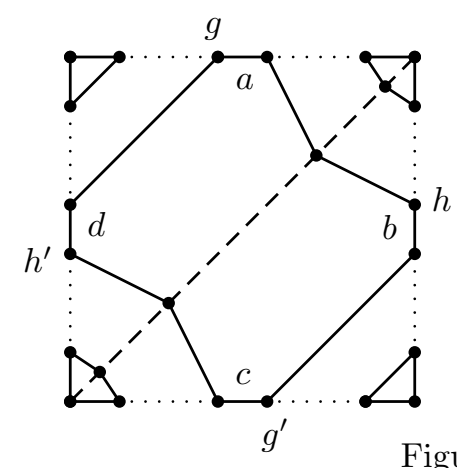

Figure 6

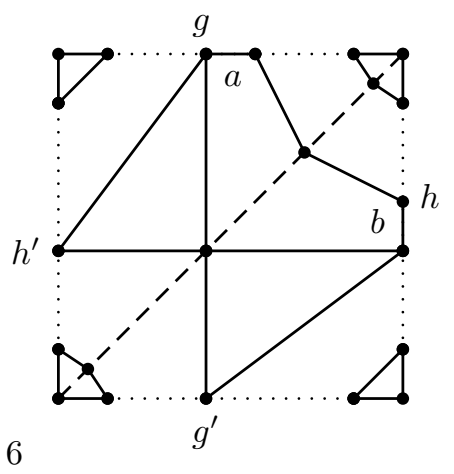

In the left hand quadrilateral in Figure 6 , the two triangles $\Delta$ and $\Delta^{\prime}$ have central hexagons and the hexagons have a side in common. Deleting the common edge $L$ of $\Delta$ and $\Delta^{\prime}$ (and so, in particular, removing the common side of the central hexagons), we see that we are left with a quadrilateral with a central octagon.

In the right hand quadrilateral of Figure $6, \Delta$ and $\Delta^{\prime}$ both have central triangles which meet at a common point. This is the only configuration in which possibility $(v)$ above holds, that is, the only one in which there is a group element matched with group elements on each of the other three sides.

We obtain a derivation of $u \# x \# w \# x$ by using the quadrilateral and the labels on the paths joining matching pairs of group elements on the sides of the quadrilateral. The precise derivation we get depends on which of the sixteen possible configurations is involved. We illustrate the process for obtaining the derivation when the configuration in question is that of Figure 5, and subsequently comment on what happens when we use the two configurations in Figure 6 . We adopt the notational convention that if $w$ is one of $u_{i}, y_{i}, z_{i}$ or $t_{i}$, then $X_{w}$ will be denoted by $U_{i}, Y_{i}, Z_{i}$ or $T_{i}$ respectively.

When we dispense with the common side of the two triangles in Figure 5 and label the paths we obtain the following diagram. 


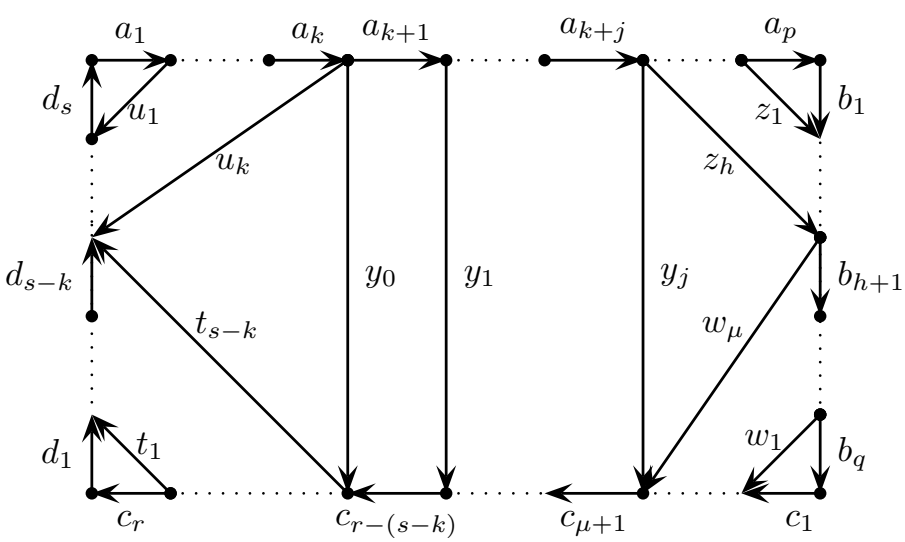

Figure 7

In this diagram, $\left(a_{1} \ldots a_{k}\right) \sigma$ is the group element on $L_{u}$ which is a vertex of the central triangle in $\Delta$. We also have $h=p-(k+j)$ and $\mu=q-h$.

We use the diagram to get a derivation in the following way. We start with the production $X_{\epsilon} \rightarrow a_{1} U_{1} d_{s}$ and then apply productions determined by internal quadrilaterals to obtain the derivation:

$$
X_{\epsilon} \Rightarrow a_{1} U_{1} d_{s} \Rightarrow a_{1} a_{2} U_{2} d_{s-1} d_{s} \Rightarrow \cdots \Rightarrow a_{1} \ldots a_{k} U_{k} d_{s-(k-1)} \ldots d_{s} .
$$

Now, corresponding to the internal triangle labelled by $y_{0}, t_{s-k}$ and $u_{k}$, apply the production $U_{k} \rightarrow Y_{0} T_{s-k}$. From $Y_{0}$, by applying productions determined by internal quadrilaterals, we obtain

$$
Y_{0} \Rightarrow a_{k+1} Y_{1} c_{r-(s-k)} \Rightarrow \cdots \Rightarrow a_{k+1} \ldots a_{k+j} Y_{j} c_{\mu+1} \ldots c_{r-(s-k)},
$$

and from $T_{s-k}$, by applying productions determined by internal quadrilaterals and the triangle labelled by $t_{1}, c_{r}$ and $d_{1}$ we get

$$
\begin{aligned}
T_{s-k} & \Rightarrow c_{r+1-(s-k)} T_{s-(k+1)} d_{s-k} \Rightarrow \cdots \Rightarrow c_{r+1-(s-k)} \ldots c_{r-1} T_{1} d_{2} \ldots d_{s-k} \\
& \Rightarrow c_{r+1-(s-k)} \ldots c_{r} \# d_{1} \ldots d_{s-k} .
\end{aligned}
$$

Now we apply the production $Y_{j} \rightarrow Z_{h} W_{\mu}$ and in a similar way obtain derivations

$$
W_{\mu} \Rightarrow b_{h+1} W_{\mu-1} c_{\mu} \Rightarrow \cdots \Rightarrow b_{h+1} \ldots b_{q} \# c_{1} \ldots c_{\mu}
$$

and

$$
Z_{h} \Rightarrow a_{k+j+1} Z_{h-1} b_{h} \Rightarrow \cdots \Rightarrow a_{k+j+1} \ldots a_{p} \# b_{1} \ldots b_{h}
$$


Putting all this together we see that

$$
X_{\epsilon} \stackrel{*}{\Rightarrow} a_{1} \ldots a_{p} \# b_{1} \ldots b_{q} \# c_{1} \ldots c_{r} \# d_{1} \ldots d_{s}
$$

as desired.

The other configurations give rise to corresponding derivations in a similar way. As we have noted, when we dispense with the common side of the the two triangles in the left hand diagram in Figure 6 there is a central octagon. If the labels of the paths joining $g$ to $h^{\prime}(d \sigma), g(a \sigma)$ to $h, h(b \sigma)$ to $g^{\prime}$ and $g^{\prime} c$ to $h^{\prime}$ are $u, y, w$ and $t$ respectively, we need a production of the form $U \rightarrow a Z b W c T d$. This explains why we need to allow words of length up to 7 on the right hand side of productions.

In the right hand diagram in Figure 6, there are group elements matched with others on the opposite side of the quadrilateral for both pairs of opposite sides. We do not use the path joining $h$ and $h^{\prime}$ in constructing the derivation from this configuration. If the labels of the paths joining $g$ to $h^{\prime}$, $g$ to $g^{\prime}, g^{\prime}$ to $h^{\prime}, g(a \sigma)$ to $h$ and $h(b \sigma)$ to $g^{\prime}$ are $u, y, t, z$ and $w$ respectively, we use productions $U \rightarrow Y T$ and $Y \rightarrow a Z b W$ as well as ones arising from internal quadrilaterals and triangles as in the above derivations.

Thus we obtain $Q \subseteq L(\mathscr{G})$ as required.

We will actually use the following easy consequence of the lemma.

Corollary 4.1. Let $\sigma: A^{*} \rightarrow G$ be a symmetric finite choice of generators for a hyperbolic group $G$, and let $P$ be the language of geodesics. Let $g$ be an element of $G$. Then the language

$$
T_{g}=\{u \# w \# x: u, w, x \in P \text { and }(u \sigma) g(w x) \sigma=1\}
$$

is context-free.

Proof. First, note that by Corollary $2.1(1), g \sigma^{-1} \cap P$ is rational so that

$$
P_{g}=P\{\#\}\left(g \sigma^{-1} \cap P\right)\{\#\} P\{\#\} P
$$

is also rational. Hence by Corollary 2.1(2), the language $Q_{g}=Q \cap P_{g}$ is context-free.

We define a finite automaton $M$ over the monoid $(A \cup\{\#\})^{*} \times(A \cup\{\#\})^{*}$. The state set of $M$ is $\left\{q_{0}, q_{1}, q_{2}\right\}$ with initial state $q_{0}$ and unique accept state $q_{2}$. The edge set is

$$
\begin{aligned}
& \left\{\left(q_{0},(a, a), q_{0}\right): a \in A\right\} \cup\left\{\left(q_{0},(\#, \epsilon), q_{1}\right)\right\} \\
& \quad \cup\left\{\left(q_{1},(a, \epsilon), q_{1}\right): a \in A\right\} \cup\left\{\left(q_{1},(\#, \#), q_{2}\right)\right\} \\
& \cup\left\{\left(\left(q_{2},(a, a), q_{2}\right): a \in A\right\} \cup\left\{\left(q_{2},(\#, \#), q_{2}\right)\right\},\right.
\end{aligned}
$$


where again we use $\epsilon$ to denote the empty word. We illustrate $M$ in Figure 8 where we use the convention that if an arrow has several labels, then it is labelled by the set of those labels. We denote the diagonal $\{(a, a): a \in A\}$ by $D$, and write $B$ for $D \cup\{\#, \#\}$.

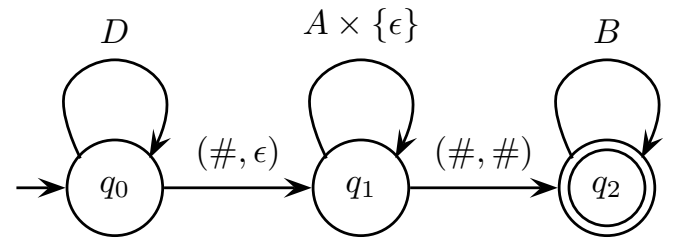

Figure 8

Let $\theta$ be the rational transduction accepted by $M$. Then, by (4) of Proposition 2.2, $Q_{g} \theta$ is context-free. But $Q_{g} \theta=T_{g}$.

We are now ready to complete the proof of Theorem 4.1 which is a consequence of the next result. We revert to the notation introduced at the beginning of the section for the $\mathscr{R}$-classes, etc. of a finitely generated completely simple semigroup $S$. In particular, we have $\mathscr{H}$-classes $H_{i j}$ for $i \in\{1, \ldots, m\}$ and $j \in\{1, \ldots, n\}$, we write $H$ for $H_{11}$, the idempotent in $H_{i j}$ is $e_{i j}, X \subseteq H$ is a symmetric finite set of generators for $H$ and $e=e_{11} \in H$.

For clarity, we let $A, B=\left\{b_{i}: i=1, \ldots, m\right\}, C=\left\{c_{j}: j=1, \ldots, n\right\}$ be disjoint sets and $\varphi: A \rightarrow X$ be a bijection. Then, extending $\varphi$ to a homomorphism $\sigma: A^{*} \rightarrow H$ gives a symmetric choice of generators for $H$, and extending $\varphi$ to $\rho:(A \cup B \cup C)^{+} \rightarrow S$ by defining $b_{i} \rho=e_{i 1}$ and $c_{j} \rho=e_{1 j}$ gives a choice of (semigroup) generators for $S$.

Proposition 4.3. Let $S$ be a finitely generated completely simple semigroup with a maximal subgroup $H$. If $H$ is hyperbolic, then $S$ is hyperbolic.

Proof. Using the above notation, let $P$ be the language of geodesics for $H$ relative to $\sigma$. Let $h_{j i}=e_{1 j} e_{i 1}$ and note that $h_{j i} \in H$. Then, by Corollary 4.1, the language

$$
T_{j i}=\left\{u \# w \# x: u, w, x \in P \text { and }(u \sigma) h_{j i}(w x) \sigma=1\right\}
$$

is context-free. Since $X$ (and so also $A$ ) is symmetric, we have $x \in P$ if and only if $x^{-1} \in P$. Thus

$$
T_{j i}=\left\{u \# w \# x^{-1}: u, w, x \in P \text { and }(u \sigma) h_{j i}(w \sigma)=x \sigma\right\} .
$$

Define a finite automaton $M$ over the semigroup $(A \cup\{\#\})^{+} \times(A \cup\{\#\})^{+}$ as follows: the state set is $\left\{q_{0}, q_{1}, q_{2}\right\}$ where $q_{0}$ is the initial state and $q_{2}$ is 
the unique accept state, and the edge set is

$$
\begin{aligned}
& \left\{\left(q_{0},(a, a), q_{0}\right): a \in A\right\} \cup\left\{\left(q_{0},(\#, \#), q_{1}\right)\right\} \\
& \quad \cup\left\{\left(q_{1},(a, a), q_{1}\right): a \in A\right\} \cup\left\{\left(q_{1},(\#, \#), q_{2}\right)\right\} \\
& \quad \cup\left\{\left(\left(q_{2},\left(a^{-1}, a\right), q_{2}\right): a \in A\right\} .\right.
\end{aligned}
$$

The automaton is illustrated in Figure 9 where $D$ denotes the diagonal $\{(a, a): a \in A\}$ and $C$ denotes the set $\left\{\left(a^{-1}, a\right): a \in A\right\}$.

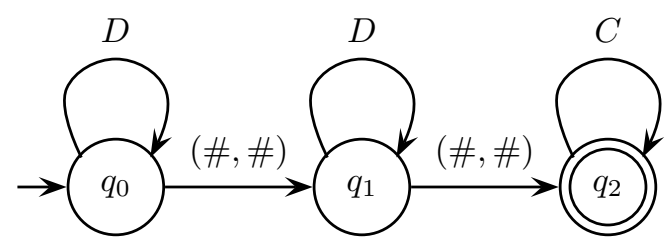

Figure 9

Let $\theta$ be the rational transduction accepted by $M$. Then, by (4) of Proposition $2.2, T_{j i} \theta$ is context-free, that is,

$$
T_{j i}^{\prime}=\left\{u \# w \# x^{r}: u, w, x \in P \text { and }(u \sigma) h_{j i}(w \sigma)=x \sigma\right\}
$$

is context-free.

We have already pointed out that $\rho:(A \cup B \cup C)^{+} \rightarrow S$ is surjective. In fact, every element of $S$ can be written as $e_{i 1} h e_{1 j}$ for some $i, j$ and some $h \in H$ (see, for example, Chapter 3 of [18]). Hence $S=L \rho$ where $L=B P C$. Clearly, $L$ is a rational subset of $(A \cup B \cup C)^{+}$, so to show that $S$ is hyperbolic, it is enough to show that the multiplication table $T_{L}$ of $S$ relative to $(A \cup B \cup C, L, \rho)$ is context-free.

Let $X=A \cup B \cup C \cup\{\#\}$. For $i, k \in\{1, \ldots, m\}$ and $j, \ell \in\{1, \ldots, n\}$, we define an automaton $M_{i j k \ell}$ over the monoid $X^{*} \times X^{*}$ as follows. There are five states $q_{0}, \ldots, q_{4}$ with $q_{0}$ being the initial state and $q_{4}$ the unique accept state. The edge set is

$$
\begin{aligned}
& \left\{\left(q_{0},\left(1, b_{i}\right), q_{1}\right)\right\} \cup\left\{\left(q_{1},(a, a), q_{1}\right): a \in A\right\} \\
& \cup\left\{\left(q_{1},\left(\#, c_{j} \# b_{k}\right), q_{2}\right)\right\} \cup\left\{\left(q_{2},(a, a), q_{2}\right): a \in A\right\} \\
& \cup\left\{\left(q_{2},\left(\#, c_{\ell} \# c_{\ell}\right), q_{3}\right)\right\} \cup\left\{\left(q_{3},(a, a), q_{3}\right): a \in A\right\} \\
& \cup\left\{\left(q_{3},\left(1, b_{i}\right), q_{4}\right)\right\} .
\end{aligned}
$$

The automaton is illustrated in Figure 10 where, as before, $D$ denotes the diagonal $\{(a, a): a \in A\}$. 


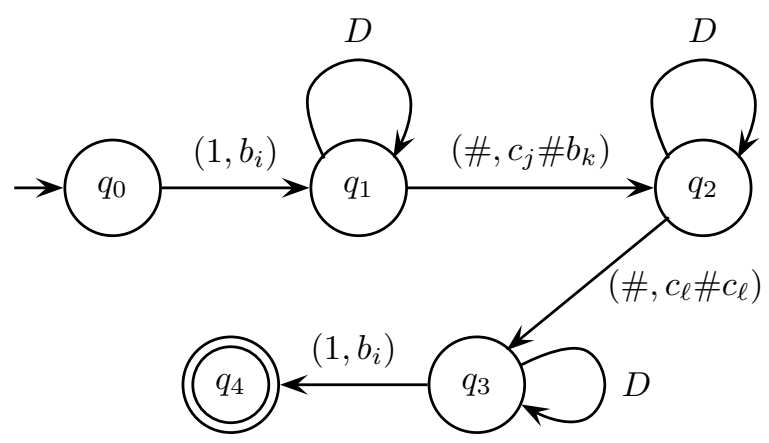

Figure 10

Let $\psi_{i j k \ell}$ be the rational transduction accepted by $M_{i j k \ell}$, and put $T_{i j k \ell}=T_{j k}^{\prime} \psi_{i j k \ell}$. Then

$$
T_{i j k \ell}=\left\{b_{i} u c_{j} \# b_{k} w c_{\ell} \#\left(b_{i} x c_{\ell}\right)^{r}: u, w, x \in P \text { and }(u \sigma) h_{j k}(w \sigma)=x \sigma\right\} \text {, }
$$

and by Proposition 2.2(4), $T_{i j k \ell}$ is context-free.

Let $\alpha, \beta, \gamma \in(A \cup B \cup C)^{+}$. Then $\alpha \# \beta \# \gamma^{r} \in T_{L}$ if and only if for some $i, k \in\{1, \ldots, m\}, j, \ell \in\{1, \ldots, n\}$ and $u, w, x \in P$ we have $\alpha=b_{i} u c_{j}$, $\beta=b_{k} w c_{\ell}, \gamma=b_{i} x c_{\ell}$ and

$$
\left(b_{i} u c_{j}\right) \rho\left(b_{k} w c_{\ell}\right) \rho=\left(b_{i} x c_{\ell}\right) \rho,
$$

that is,

$$
e_{i 1}(u \sigma) h_{j k}(w \sigma) e_{1 \ell}=\left(e_{i 1}(u \sigma) e_{1 j}\right)\left(e_{k 1}(w \sigma) e_{1 \ell}\right)=e_{i 1}(x \sigma) e_{1 \ell} .
$$

The latter holds if and only if $(u \sigma) h_{j k}(w \sigma)=x \sigma$. Hence $T_{L}=\bigcup T_{i j k \ell}$ where the indices $i, k$ range over $1, \ldots, m$ and $j, \ell$ range over $1, \ldots, n$. Thus $T_{L}$ is a finite union of context-free languages, and so is itself context-free as required.

We conclude with a straightforward corollary of Theorem 4.1. First, we recall the notion of an 'automatic semigroup'. Let $A$ be a finite alphabet and $\$$ be a symbol not in $A$. We define a function $\delta:\left(A^{+} \times A^{+}\right) \rightarrow\left(A^{\$} \times A^{\$}\right)^{+}$ where $A^{\$}=A \cup\{\$\}$ by

$$
(v, w) \delta= \begin{cases}\left(a_{1}, b_{1}\right) \ldots\left(a_{m}, b_{m}\right) & \text { if } m=n \\ \left(a_{1}, b_{1}\right) \ldots\left(a_{m}, b_{m}\right)\left(\$, b_{m+1}\right) \ldots\left(\$, b_{n}\right) & \text { if } m<n \\ \left(a_{1}, b_{1}\right) \ldots\left(a_{n}, b_{n}\right)\left(a_{n+1}, \$\right) \ldots\left(a_{m}, \$\right) & \text { if } m>n\end{cases}
$$

where $v=a_{1} \ldots a_{m}$ and $w=b_{1} \ldots b_{n}$.

Let $\varphi: A^{+} \rightarrow S$ be a finite choice of generators for the semigroup $S$. A triple $(A, L, \varphi)$ is an automatic structure for $S$ if $L$ is a rational subset of 
$A^{+}$with $L \varphi=S$ such that the sets

$$
L_{=}=\{(v, w) \delta: v, w \in L \text { and } v \varphi=w \varphi\}
$$

and

$$
L_{a}=\{(v, w) \delta: v, w \in L \text { and }(v a) \varphi=w \varphi\}
$$

are rational subsets of $\left(A^{\$} \times A^{\$}\right)^{+}$for every $a \in A$. A semigroup is automatic if it has an automatic structure, and it is prefix-automatic if it has an automatic structure $(A, L, \varphi)$ with $L$ prefix-closed. We remark that this definition is equivalent to that used in [23], [24] (see, for example, Proposition 7.1 of [19]).

It follows from Theorem 2.5.9 of [10] that an automatic group is prefixautomatic. However, whether or not every automatic semigroup is prefixautomatic is an open question.

Examples of hyperbolic monoids which are not automatic are given in [16], but it is known that every hyperbolic group is automatic (see, for example Corollary 2.20 of Chapter III. $\Gamma$ in [4]). For completely simple semigroups, the situation is similar to that for groups. First, we quote part of Theorem 7.9 of [19].

Proposition 4.4. Let $M$ be a finitely generated Rees matrix semigroup (without zero) over a group $G$. Then $M$ is prefix-automatic if and only if $G$ is automatic.

Corollary 4.2. Every hyperbolic completely simple semigroup is prefixautomatic.

Proof. Let $S$ be a hyperbolic completely simple semigroup. Then $S$ is finitely generated and isomorphic to a Rees matrix semigroup $M(G ; I, J ; P)$. By Theorem 4.1, $G$ is hyperbolic, and hence, by the results quoted above, $G$ is prefix-automatic. Now, by Proposition $4.4, S$ is prefix-automatic.

\section{Acknowledgements}

The first author would like to thank the organisers (M. Branco, V. H. Fernandes and Gracinda M. S. Gomes) for the invitation to speak at the Semigroups and Languages workshop.

The work was carried out while the second author was a research student at the University of York supported by an EPSRC studentship. He would like to thank Kirsty for all her support and encouragement. 


\section{References}

1. J. Alonso, T. Brady, D. Cooper, V. Ferlini, M. Lustig, M. Mihalik, M. Shapiro and H. Short, Notes on word hyperbolic groups, in Group Theory from a Geometrical Viewpoint (E. Ghys, A. Haefliger and A. Verjovsky, eds.), World Scientific, (1991), 3-63.

2. H. Ayik and N. Ruškuc, Generators and relations of Rees matrix semigroups, Proc. Edinburgh Math. Soc., 42 (1999), 481-495.

3. J. Berstel, Transductions and context-free languages, Teubner, 1979.

4. M. Bridson and A. Haefliger, Metric spaces of non-positive curvature, Springer-Verlag, 1999.

5. W. W. Boone, The word problem, Ann. of Math., 2 (1959), 207-265.

6. J. L. Britton, The word problem for groups, Proc. London Math. Soc., 8 (1958), 493-506.

7. M. Dehn, Über unendliche diskontinuierliche Gruppen, Math. Ann. 71 (1912), 116-144.

8. M. Dehn, Transformationen der Kurven auf zweitseitigen Flächen, Math. Ann. 72 (1912), 413-421.

9. A. J. Duncan and R. H. Gilman, Word hyperbolic semigroups, Math. Proc. Camb. Phil. Soc., to appear.

10. D. B. A. Epstein et al., Word processing in groups, Jones and Bartlett, 1992.

11. E. Ghys and P. de la Harpe (eds.), Sur les groupes hyperboliques d'apres Mikhael Gromov, Birkhäuser, 1990.

12. R. H. Gilman, Formal languages and infinite groups, in Geometrical and Computational Perspectives on Infinite Groups. DIMACS Ser. Discrete Math. Theoret. Comput. Sci. 25, Amer. Math. Soc., (1996), 27-51.

13. R. H. Gilman, On the definition of word hyperbolic groups, Math. Zeitschr., 242 (2002), 529-541.

14. M. Gromov, Hyperbolic groups, in Essays in Group Theory (S. M. Gersten, ed.), Springer-Verlag, MSRI Publ. 8 (1987), 75-263.

15. M. Harrison, Introduction to Formal Language Theory, Addison-Wesley, 1978.

16. M. Hoffmann, D. Kuske, F. Otto and R. M. Thomas, Some relatives of automatic and hyperbolic groups, Proc. of the Thematic Term on Semigroups, Automata, Algorithms and Languages (G. M. S. Gomes, J.-E. Pin and P. V. Silva, eds.), World Scientific, (2002), 379-406.

17. J. E. Hopcroft and J. D. Ullman, Introduction to Automata Theory, Languages and Computation, Addison-Wesley, 1979.

18. J. M. Howie, Fundamentals of Semigroup Theory, Oxford University Press, 1995)

19. M. E. Kambites, Combinatorial aspects of partial algebras, Ph.D. thesis, University of York, 2003.

20. R. C. Lyndon and P. E. Schupp, Combinatorial group theory, Springer-Verlag, 1977.

21. P. S. Novikov, On the algorithmic unsolvability of the word problem in group theory, (Russian), Trudy Mat. Inst. Steklov, 44 (1955), 143 pp. 
22. K. Ohshika, Discrete groups, Amer. Math. Soc., 2002.

23. P. V. Silva and B. Steinberg, Extensions and submonoids of automatic monoids, Theor. Comp. Sci. 289 (2002), 727-754.

24. P. V. Silva and B. Steinberg, A geometric characterization of automatic monoids Quart. J. Math., to appear.

25. B. Steinberg, A topological approach to inverse and regular semigroups, $P a$ cific J. Math. 208 (2003), 367-396.

26. J. B. Stephen, Presentations of inverse monoids, J. Pure Appl. Algebra 63 (1990), 81-112. 